\title{
QUANTIFYING OCEAN ACOUSTIC ENVIRONMENTAL SENSITIVITY
}

\author{
Stan E. Dosso ${ }^{1}$, Diana F. McCammon ${ }^{2}$, Peter M. Giles ${ }^{3}$, Sean Pecknold ${ }^{4}$, and Paul C. Hines ${ }^{4}$ \\ ${ }^{1}$ School of Earth and Ocean Sciences, University of Victoria, Victoria, B.C. Canada, V8W 3P6 \\ ${ }^{2}$ McCammon Acoustical Consulting, 475 Baseline Rd, Waterville, NS Canada, B0P 1P0 \\ ${ }^{3}$ General Dynamics Canada, 11 Thornhill Dr., Dartmouth, NS Canada, B3B 1R9 \\ ${ }^{4}$ Defence Research and Development Canada-Atlantic, Dartmouth, NS Canada, B2Y $3 Z 7$
}

\section{INTRODUCTION}

This paper defines and examines linear, linearized, and nonlinear measures of environmental sensitivity for ocean acoustic propagation that account for realistic uncertainties in various environmental parameters (watercolumn sound-speed profile and seabed geoacoustic properties). Simple interpretations of sensitivity are typically based on the implicit assumption of a linear relationship between parameter sensitivity and parameter uncertainty. This assumption is examined by comparing the three sensitivity measures over a range of parameter uncertainties about the actual assumed environmental uncertainty. Sensitivity range and depth dependencies are illustrated for realistic geoacoustic uncertainties and oceanographic variability of the sound-speed profile.

\section{THEORY}

The interpretation of sensitivity is well defined for linear problems, which provide a basis for addressing nonlinear problems such as ocean acoustic propagation. For a linear problem, the change in datum $d_{i}$ due a change $\delta m_{j}$ to the $j$ th environmental model parameter $m_{j}$ is

$$
\delta d_{i}^{j}=d_{i}\left(m_{j}+\delta m_{j}\right)-d_{i}\left(m_{j}\right)=\frac{\partial d_{i}}{\partial m_{j}} \delta m_{j} .
$$

Equation (1) indicates how uncertainties transfer from parameters to data for a linear problem: If the uncertainty for parameter $m_{j}$ is Gaussian distributed with standard deviation $\sigma_{j}$, then the corresponding uncertainty for datum $d_{i}$ is also Gaussian with standard deviation $\partial d_{i} / \partial m_{j} \sigma_{j}$; i.e., sensitivities scale directly with parameter uncertainties. A linear sensitivity measure that is independent of data/parameter scales and units is defined

$S_{i j}=\frac{\left|\delta d_{i}\left(\delta m_{j}=\sigma_{j}\right)\right|}{\left|d_{i}\right|}$.

Equation (2) represents the ratio of the standard deviation of the $i$ th datum to its expected value and is equivalent to the coefficient of variation, a quantity commonly used for comparing the variability of potentially disparate quantities.
The above concepts can be extended to weakly nonlinear problems. Expanding the data functional for a parameter perturbation about the background model and neglecting second-order terms leads to an approximate local linear relationship between data and parameter perturbations which can be used in a linearized sensitivity measure; for improved accuracy, a two-sided average can be defined

$$
S_{i j}=\frac{1}{2}\left[\frac{\left|d_{i}\left(m_{j}+\delta \sigma_{j}\right)-d_{i}\left(m_{j}\right)\right|}{\left|d_{j}\right|}+\frac{\left|d_{i}\left(m_{j}-\delta \sigma_{j}\right)-d_{i}\left(m_{j}\right)\right|}{\left|d_{j}\right|}\right]
$$

To address nonlinearity explicitly, the full data uncertainty distribution can be sampled using a Monte Carlo approach to draw random model perturbations delta $m_{j}$ from a Gaussian distribution with zero mean and standard deviation $\sigma_{j}$, and computing the corresponding data perturbation for each sample. A nonlinear sensitivity measure is based on the root-mean-square average perturbation

$S_{i j}=\frac{\left[\left\langle d_{i}\left(m_{j}+\delta m_{j}\right)-\left.d_{i}\left(m_{j}\right)\right|^{2}\right\rangle\right]^{1 / 2}}{\left|d_{i}\right|}$

For a linear problem, the linear, linearized, and nonlinear sensitivity measures are identical. Due to the highly variable nature of propagating acoustic fields, data sensitivities must be spatially averaged, as indicated in the following section, to obtain a stable, representative sensitivity measure [1].

The linearity of parameter sensitivities can be examined by comparing the linear, linearized, and nonlinear sensitivity measures over a range of parameter standard deviations $\sigma_{j}$ about the actual environmental uncertainty.

\section{EXAMPLES}

This section provides examples of the sensitivity analysis outlined above. The environmental parameters and uncertainties are based on the Malta Plateau, a well-studied region of the Mediterranean Sea. The environmental model, illustrated in Fig. 1, is comprised of a 131-m water column 


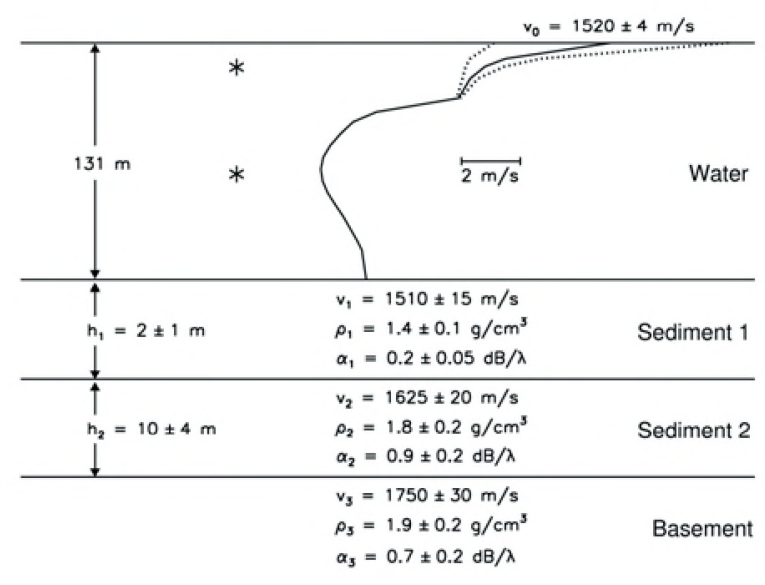

Fig. 1. Malta Plateau environment, including assumed values and standard deviations for environmental parameters. Asterisks indicate source depths.

over a three-layer seabed. The ocean sound-speed profile (SSP) includes a strong negative gradient in the near-surface waters and a weak sound channel with its axis near midwater depth. The three seabed layers are characterized by sound speeds $v_{1}, v_{2}, v_{3}$, densities $\rho_{1}, \rho_{2}, \rho_{3}$, and attenuation coefficients $\alpha_{1}, \alpha_{2}, \alpha_{3}$. The upper two sediment layers are of thicknesses $h_{1}$ and $h_{2}$ (the basement layer is semi-infinite). Geoacoustic and SSP parameters values and uncertainties representative of the Malta Plateau region are given in Fig. 1. The SSP uncertainty is taken to represent oceanographic variability due to surface heating/cooling and wind mixing, with the effects decaying exponentially with depth over the top $30 \mathrm{~m}$, as shown in Fig. 1. This variability is represented by the standard deviation of the surface sound speed $v_{0}$.

Fig. 2 shows the three sensitivity measures for all environmental parameters averaged over $0-30 \mathrm{~m}$ receiver depth and $0-20 \mathrm{~km}$ range for source depths of 15 and $65 \mathrm{~m}$. Sensitivities for the different parameters vary over almost six orders of magnitude, with the most sensitive parameters being those of the upper seabed layer and SSP, followed by the second and then third seabed layers. The sensitivity results for the two source depths in Fig. 4 are generally similar, with the largest difference for the SSP parameter $v_{0}$. In all cases the linearized estimate provides a better approximation to the true nonlinear sensitivity measure.

Figure 3 illustrates the three sensitivities for selected parameters as a function of range and depth, with the parameter standard deviation fixed at the assumed environmental uncertainties for the Malta Plateau region (Fig. 1). For the three geoacoustic parameters $v_{1}, v_{2}$, and $h_{1}$, the source is at $65-\mathrm{m}$ depth near the sound-channel axis, while for the SSP parameter $v_{0}$, the source is at $15-\mathrm{m}$ depth within the variable near-surface layer. The sensitivity to the second sediment layer sound speed $v_{2}$ includes high values at short ranges, as steep propagation paths (which attenuate with range) interact significantly with the deeper layer. The
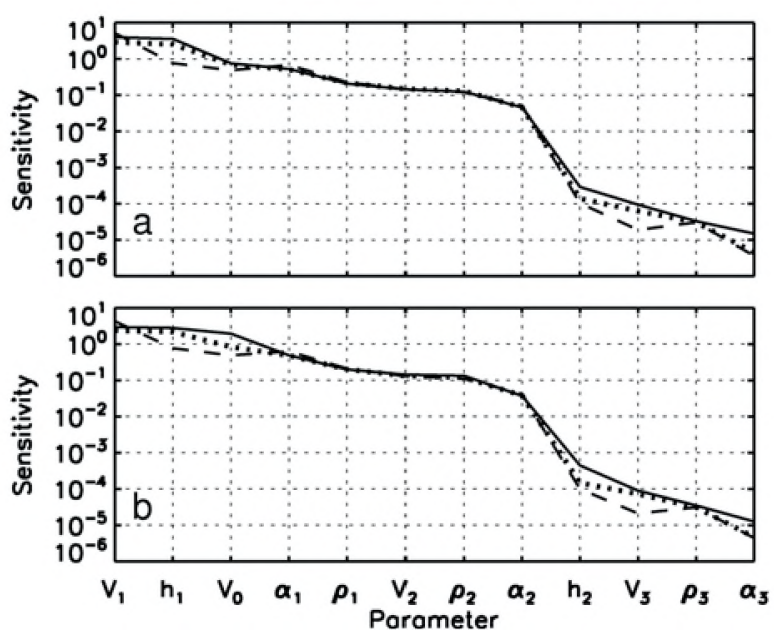

Fig. 2. Parameter sensitivities averaged over receiver depths of 0 $30 \mathrm{~m}$ for source depths of (a) $65 \mathrm{~m}$ and (b) $15 \mathrm{~m}$. Nonlinear, linearized, and linear sensitivity measures are indicated by solid, dotted, and dashed lines, respectively.

highest sensitivity for the SSP parameter $v_{0}$ is confined to depths over which the SSP varies. The agreement of the linearized and linear sensitivities with the nonlinear sensitivity in Fig. 3 is good for parameter $v_{1}$ and excellent for $v_{2}$, with generally better agreement for the linearized measure. The linearized sensitivity is in reasonable agreement with the nonlinear sensitivity for parameter $h_{1}$, but in poor agreement for $v_{0}$. The linear sensitivity differs substantially from the nonlinear sensitivity for $h_{1}$ and $v_{0}$.

\section{REFERENCES}

[1] R. T. Kessel, "A mode-based measure of field sensitivity to geoacoustic parameters in weakly range-dependent environments," J. Acoust. Soc. Am., 105, 122-129 (1999).
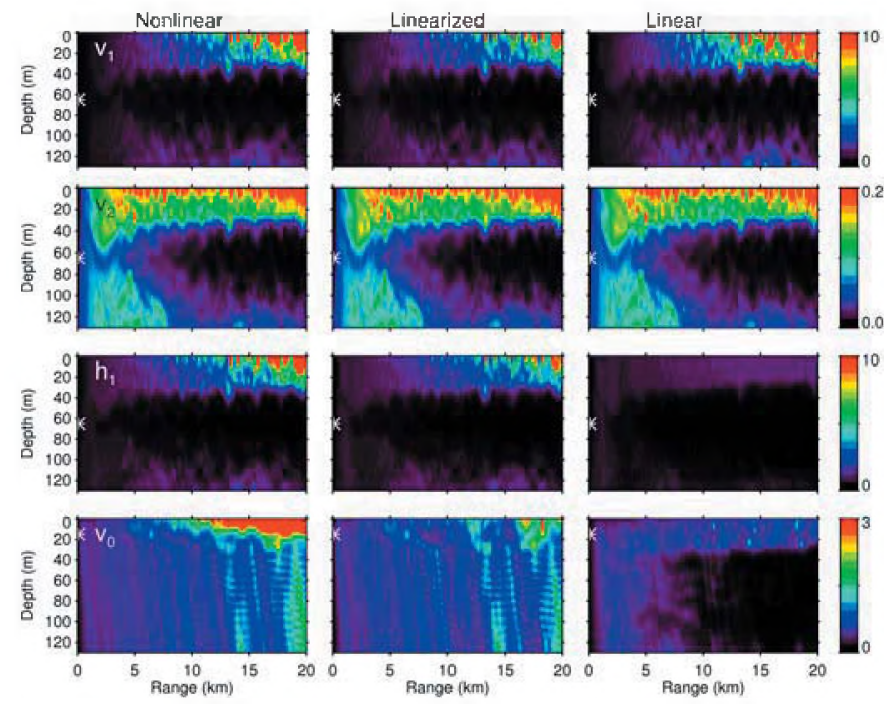

Fig. 3. Sensitivity sections for parameters $v_{1}, v_{2}, h_{1}$, and $v_{0}$ (rows) and nonlinear, linearized, and linear sensitivity measures (columns). Source depth indicated by asterisks. 